\title{
Joint SDMA and Power-Domain NOMA System for Multi-User Mm-Wave Communications
}

\author{
Israa Khaled $^{* \dagger}$, Charlotte Langlais*, Ammar El Falou ${ }^{\dagger}$, Michel Jezequel*, Bachar ElHasssan ${ }^{\dagger}$ \\ * IMT Atlantique, Electronics Department, CNRS UMR 6285 Lab-STICC, Brest, France \\ $\dagger$ Lebanese University, Faculty of Engineering, Tripoli, Lebanon
}

\begin{abstract}
Digital beamsteering (DBS) is an appealing technique to overcome the severe millimeter-wave path loss, with less complexity and less channel feedback. In multi-user systems, DBS generates a beam toward each user. However, in congested cells, such a system suffers from high inter-user interference causing by the neighboring beams. In this paper, a new approach of space division multiple access (SDMA) with power-domain non-orthogonal multiple access (PD-NOMA) is considered for millimeter-wave channel to significantly reduce the inter-user interference and thus enhance the overall throughput. The proposed scheme is designed by selecting the users located at the same direction to share one beam, in which PD-NOMA multiplexes the users in power domain. The proposed user clustering method built upon an interference metric, and followed by a fixed power allocation method based on the received power, makes possible the design of a low-feedback NOMA-DBS scheme. Simulation results, based on the New York university mm-wave simulator, show that the multi-user MIMO system can achieve higher sum-rate with our proposed joint SDMA and PD-NOMA scheme, with respect to only SDMA (e.g., up to $20 \mathrm{bps} / \mathrm{Hz}$ rate gain for 45 users in the cell).
\end{abstract}

Index Terms-Beamforming, multi-user MIMO, millimeterwave, antenna array, power-domain NOMA.

\section{INTRODUCTION}

In millimeter-wave (mmWave) wireless communications, digital beamforming (BF) with high directional array is investigated as an attractive technique to mitigate the tremendous path loss and achieve high data rates [1], [2]. Specifically, in a multi-user system, digital beamsteering (DBS) is one of the promising beamforming techniques in terms of complexity and channel feedback [3], [4]. Based on a geometric information knowledge at the transmitter (TX), mainly the angle of the line-of sight (LOS) path, DBS forms and steers a beam toward the desired user using digital phase shifters, achieving spatial division multiple access (SDMA).

Recently, non-orthogonal multiple access (NOMA) has been investigated with mmWave massive multiple-input multipleoutput (m-MIMO) systems in order to further increase the spectrum efficiency, by exploiting the multiplexing in the power-domain (PD) [5], [6]. Different NOMA-based multiuser BF (NOMA-BF) system was proposed in the literature to support multiple users per a single $\mathrm{BF}$ vector, and thus enhance the overall capacity [7]-[11]. Most of them have used zero forcing $(\mathrm{ZF})$ as the $\mathrm{BF}$ technique to cancel the intercluster interference [8], [9]. However, $\mathrm{ZF} B F$ is difficult to implement in practice due to the complexity of the matrix inversion implementation. Furthermore, most of existing works on user clustering and power allocation for NOMA-BF system are designed based on a full channel state information at the transmitter [9]-[11]. A m-MIMO-NOMA system with limited feedback is designed in [12], where m-MIMO-NOMA system is decomposed, thanks to singular value decomposition, into multiple SISO-NOMA channels, by exploiting the spatial correlation matrices of users' channels.

In our previous work [4], [13], we investigated DBS in mmWave systems using stochastic mmWave channel model based on real measurements, called NYUSIM, developed at New York university [14]. We find that DBS is an appealing beamformer in terms of complexity and channel overhead. Unfortunately, in a congested cell, the MU-MIMO system suffers from high inter-user interference causing by the adjacent beams pointed toward users located at the same direction, and therefore the DBS performance degrades, compared to conjugate beamforming (CB) [15].

In this paper, we aim to design a scheme in downlink multi-user system, which enhances the DBS performance by reducing the inter-user interference. In our proposed scheme, space division multiple access (SDMA) realized by DBS is coupled with PD-NOMA, where the users located at the same direction share the same beam and are multiplexed into power domain using PD-NOMA transmission protocol. At the receivers, successive interference cancellation (SIC) is applied to decode the signals [5]. With our joint SDMA and PD-NOMA scheme, each user is served over all the bandwidth, and with only partial channel feedback, i.e., the estimated users' direction and the received power at each user. In addition to the low-complexity of DBS, we design a low-complex clustering algorithm based on a new interference geometric metric followed by a fixed power allocation method relying on the received power.

The rest of this paper is organized as follows: the MU beamforming system and problem formulation are introduced in Section II. The proposed joint SDMA and PD-NOMA strategy including the new interference metric are presented in Section III. The proposed user clustering algorithm and power allocation method are discussed in Section IV, in addition to the analysis of the sum-rate in mono-path channel. Simulation results are shown in Section V, while conclusions and perspectives are finally drawn in Section VI.

Symbol Notations: A is a matrix, a is a vector, $a$ is a scalar, (. $)^{T}$ and $(.)^{H}$ stand for the transpose and the conjugate transpose, respectively. $\mathcal{N}\left(\mu, \sigma^{2}\right)$ denotes a Gaussian random vector with mean $\mu$ and variance $\left.\sigma^{2} . \mathbf{A}_{(} i, j\right)$ is the $(i, j)$ th 
element of $\mathbf{A} \cdot \operatorname{Tr}(\cdot)$ denotes the trace of a matrix.

\section{Multi-User Beamforming System And Problem FORMULATION}

\section{A. System Model}

We consider a downlink multi-user beamforming system, in which a single base station (BS) equipped with $M$ transmit antennas serves $K$ single-antenna user equipments (UEs) $(K<M)$. BS applies the DBS beamforming, and thereby simultaneously generates $K$ beams, each beam serves one UE. Therefore, the received signal $\mathbf{y} \in \mathbb{C}^{K \times 1}$ can be expressed as:

$$
\mathbf{y}=\sqrt{\eta} \mathbf{H W s}+\mathbf{n}
$$

where $\mathbf{s} \in \mathbb{C}^{K \times 1}$ contains the data symbol of all UEs, $\mathbf{W}=\left[\begin{array}{lll}\mathbf{a}_{1} & \cdots & \mathbf{a}_{K}\end{array}\right] \in \mathbb{C}^{M \times K}$ is the DBS beamforming matrix, with $\mathbf{a}_{k}=\mathbf{a}\left(\theta_{k}\right) \in \mathbb{C}^{M \times 1}$ the array steering vector corresponding to the direction $\theta_{k}$ of the $k$ th $\mathrm{UE}\left(\mathrm{UE}_{k}\right)$, $\mathbf{H}=\left[\begin{array}{lll}\mathbf{h}_{1}^{T} & \cdots & \mathbf{h}_{K}^{T}\end{array}\right]^{T} \in \mathbb{C}^{K \times M}$ is the MU-MIMO channel matrix with $\mathbf{h}_{k} \in \mathbb{C}^{1 \times M}$ the channel of $\mathrm{UE}_{k}$, which is assumed to be a mono-path and LOS channel $\left(\mathbf{h}_{k}=\alpha_{k} \mathbf{a}_{k}^{H}\right.$, with $\alpha_{k}$ the complex gain), $\eta=\frac{1}{\operatorname{Tr}\left(\mathbf{W}^{H} \mathbf{W}\right)}=\frac{1}{M K}$ is the normalization factor that eliminates the beamforming effect on the transmission power, and $\mathbf{n} \in \mathbb{C}^{K \times 1}$ is the additive white Gaussian noise vector with $\mathbf{n} \sim \mathcal{N}\left(0, \sigma_{n}^{2}\right)$. For a horizontal uniform linear array (ULA) along $x$-axis, $\mathbf{a}(\theta)$ is a function of the angular direction $\theta$ of the plane wave, and is given by:

$$
\mathbf{a}(\theta)=\left[1, e^{j 2 \pi \frac{d}{\lambda} \cos (\theta)}, \cdots, e^{j 2 \pi(M-1) \frac{d}{\lambda} \cos (\theta)}\right]^{T}
$$

where $d$ is the antenna spacing and $\lambda$ is the wavelength.

\section{B. Problem Formulation}

In mono-path environment, the signal-to-interference-plusnoise ratio $\mathrm{SINR}_{k}^{\mathrm{DBS}}$ achieved with $\mathrm{DBS}$ at $\mathrm{UE}_{k}$ is given by [4]:

$$
\operatorname{SINR}_{k}^{\mathrm{DBS}}=\frac{\left|\mathbf{a}_{k}^{H} \mathbf{a}_{k}\right|^{2}}{\sum_{u=1, u \neq k}^{K}\left|\mathbf{a}_{k}^{H} \mathbf{a}_{u}\right|^{2}+\frac{\sigma_{n}^{2}}{\eta \alpha_{k}^{2}}}
$$

With a phased antenna array, DBS forms a directive beam pointing to each UE. Unfortunately, when UEs are located at the same direction, the cellular system suffers from high inter-user interference, generated by their adjacent transmitted beams, as depicted in the left side of Fig. 1. This implies a great degradation in the system throughput. Obviously, as seen at the denominator in (3), the interference at $\mathrm{UE}_{k}$ is caused by the beams generated toward other UEs ( $\mathrm{UE}_{u}, \forall u \neq k$ ) having a large $\left|\mathbf{a}_{k}^{H} \mathbf{a}_{u}\right|$.

\section{Proposed Solution:NOMA-DBS System}

To boost the performance of the digital beamsteering multiuser system, we propose an inter-beam interference reduction method by using DBS with PD-NOMA. This method is based on a new interference metric, that we will explore later in Section III-B. The basic concept is that UEs located at the same direction are regrouped in the same cluster and superimposed using PD-NOMA transmission protocol sharing one beam, as
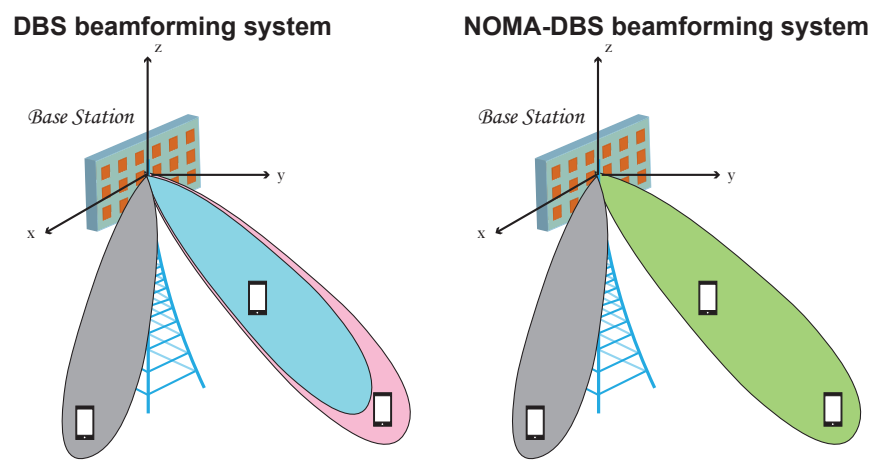

Fig. 1: DBS vs NOMA-DBS beamforming (BF) systems. depicted in the right hand-side of Fig. 1. In our proposed scheme, we consider that one or two users belong to the same cluster. However, it can be easily generalized to serve multiple users in the same cluster. The classical PD-NOMA needs full channel knowledge at the transmitter. However, DBS is chosen as an appealing beamformer, trading off complexity and channel overhead (in ULA, only the angles $\theta_{k} \forall k$ are known). Then, we design a NOMA-DBS scheme that requires partial channel knowledge; the angle direction $\theta_{k}$ and the received power $p_{k}=\left|\mathbf{h}_{k} \mathbf{w}_{k}\right|^{2}$ at $\mathrm{UE}_{k},(\forall k \in\{1 \cdots K\})$.

\section{A. NOMA-DBS System Model}

The $K$ UEs served by $K$ beams in Section II-A, are now served by $C$ beams (clusters) $(C \leq K<M)$, with $K_{c}$ UEs per cluster $\left(K_{c} \in\{1,2\}\right)$. The channel vector between BS and the $l$ th user in the $c$ th cluster, i.e, $\mathrm{UE}_{l, c} \quad\left(1 \leq l \leq K_{c}\right.$, $\left.K_{c} \in\{1,2\}, \quad c \in\{1, \cdots, C\}\right)$ denoted as $\mathbf{h}_{l, c} \in \mathbb{C}^{1 \times M}$, is given by: $\mathbf{h}_{l, c}=\alpha_{l, c} \mathbf{a}_{l, c}^{H}$ (mono-path channel assumption). Additionally, the DBS beamforming matrix will be given by $\mathbf{W}=\left[\mathbf{a}_{1} \cdots \mathbf{a}_{C}\right] \in \mathbb{C}^{M \times C}$, where $\mathbf{a}_{c}$ is the array steering vector corresponding to the direction $\theta_{c}$ of the $c$ th cluster, such that

$$
\theta_{c}= \begin{cases}\theta_{1, c} & \text { if } K_{c}=1 \\ \frac{\theta_{1, c}+\theta_{2, c}}{2} & \text { if } K_{c}=2\end{cases}
$$

This implies $\eta=\frac{1}{\operatorname{Tr}\left(\mathbf{W}^{H} \mathbf{W}\right)}=\frac{1}{M C}$. Therefore, the signals $\mathbf{x} \in \mathbb{C}^{M \times 1}$ transmitted from BS can be expressed as:

$$
\mathbf{x}=\sqrt{\eta} \mathbf{W} \mathbf{s}
$$

where $\mathbf{s} \in \mathbb{C}^{C \times 1}$ is the superimposed signal given by:

$$
\mathbf{s}=\left(\begin{array}{c}
\sum_{l=1}^{K_{1}} \sqrt{\gamma_{l, 1}} s_{l, 1} \\
\vdots \\
\sum_{l=1}^{K_{C}} \sqrt{\gamma_{l, C}} s_{l, C}
\end{array}\right)
$$

with $s_{l, c}$ the signal of $\mathrm{UE}_{l, c}$ and $\gamma_{l, c}$ its corresponding power allocation coefficient that satisfies $\sum_{k=1}^{K_{c}} \gamma_{l, c}=1, \forall c \in$ $\{1, \cdots, C\}$. For a 2 -user cluster, we denote $\mathrm{UE}_{1, c}$ the high channel gain UE (strong $\mathrm{UE}$ ) and $\mathrm{UE}_{2, c}$ the low channel gain UE (weak UE), i.e., $\left|\mathbf{h}_{1, c} \mathbf{w}_{1, c}\right|^{2} \geq\left|\mathbf{h}_{2, c} \mathbf{w}_{2, c}\right|^{2}$. PD-NOMA allocates more power to the weak user and less power to the strong one [5], i.e., $\gamma_{1, c} \leq \gamma_{2, c}$.

Then, $\mathrm{UE}_{1, c}$ applies SIC to eliminate perfectly the interference from $\mathrm{UE}_{2, c}$, while $\mathrm{UE}_{2, c}$ treats the $\mathrm{UE}_{1, c}$ signal as 
interference. Therefore, the received signals $\mathbf{y}_{1, c}$ at $\mathrm{UE}_{1, c}$ and $\mathbf{y}_{2, c}$ at $\mathrm{UE}_{2, c}$ can be written as:

$$
\mathbf{y}_{1, c}=\sqrt{\eta} \sqrt{\gamma_{1, c}} \mathbf{h}_{1, c} \mathbf{w}_{c} s_{1, c}+\sum_{\substack{b=1 \\ b \neq c}}^{C} \sum_{q=1}^{K_{b}} \sqrt{\eta} \sqrt{\gamma_{q, b}} \mathbf{h}_{1, c} \mathbf{w}_{b} s_{q, b}
$$$$
\mathbf{y}_{2, c}=\underbrace{\sqrt{\eta} \sqrt{\gamma_{2, c}} \mathbf{h}_{2, c} \mathbf{w}_{c} s_{2, c}}_{\text {desired signal }}+\underbrace{\sqrt{\eta} \sqrt{\gamma_{1, c}} \mathbf{h}_{2, c} \mathbf{w}_{c} s_{1, c}}_{\text {intra-beam interference }}
$$$$
+\underbrace{\sum_{\substack{b=1 \\ b \neq c}}^{C} \sum_{q=1}^{K_{b}} \sqrt{\eta} \sqrt{\gamma_{q, b}} \mathbf{h}_{2, c} \mathbf{w}_{b} s_{q, b}}_{\text {inter-beam interference }}
$$

\section{B. Interference Metric}

We define $\beta_{k, u}=\frac{1}{M}\left|\mathbf{a}_{k}^{H} \mathbf{a}_{u}\right|=\frac{1}{M}\left|\mathbf{a}_{u}^{H} \mathbf{a}_{k}\right|=\beta_{u, k}$ as a metric representing the interference at $\mathrm{UE}_{k}$ from the beam generated for $\mathrm{UE}_{u}$, and vice versa, with $0<\beta_{k, u} \leq 1$. For ULA, $\beta_{k, u}$ can be expressed as:

$$
\begin{aligned}
\beta_{k, u} & =\frac{1}{M}\left|\sum_{m=1}^{M} e^{j 2 \pi(m-1) \frac{d}{\lambda}\left(\cos \left(\theta_{k}\right)-\cos \left(\theta_{u}\right)\right)}\right| \\
& =\left|\frac{\sin \left(\frac{M \pi d}{\lambda}\left(\cos \left(\theta_{k}\right)-\cos \left(\theta_{u}\right)\right)\right)}{M \sin \left(\frac{\pi d}{\lambda}\left(\cos \left(\theta_{k}\right)-\cos \left(\theta_{u}\right)\right)\right)}\right|
\end{aligned}
$$

In order to regroup the users located at the same direction, we need to know the level of interference at each $\mathrm{UE}_{k}(\forall k \in$ $\{1 \cdots K\})$ from the others UEs $\left(\mathrm{UE}_{u}, \forall u \neq k\right)$. However, this level strongly depends on two factors: 1) the width $\Omega_{u}$ of the beams generated at $\mathrm{UE}_{u}(\forall u \neq k)$, which depends on the number of antennas and the beam direction, and 2) the $\mathrm{UE}_{k}$ direction with respect to $\mathrm{UE}_{u}$ direction, i.e., the angular distance $\left(\Delta \theta=\left|\theta_{k}-\theta_{u}\right|\right)$.

In order to further investigate $\beta_{k, u}$, we first define the antenna array pattern $A F(\mathbf{v}, \theta)$, such that:

$$
A F(\mathbf{v}, \theta)=\sum_{m=1}^{M}[\mathbf{v}]_{m} e^{j 2 \pi / \lambda(m-1) d \cos (\theta)}
$$

where $[\mathbf{v}]_{m}$ is the complex weight assigned to the $m$ th antenna element. However, to point a beam at $\theta_{0}$, the weights will be $[\mathbf{v}]_{m}=e^{-j 2 \pi / \lambda(m-1) d \cos \left(\theta_{0}\right)}$, and thereby (10) can be expressed as:

$$
\begin{aligned}
A F_{\theta_{0}}(\theta) & =\sum_{m=1}^{M} e^{j \frac{2 \pi d}{\lambda}(m-1)\left(\cos (\theta)-\cos \left(\theta_{0}\right)\right)} \\
& =\frac{\sin \left(\frac{M \pi d}{\lambda}\left(\cos (\theta)-\cos \left(\theta_{0}\right)\right)\right)}{\sin \left(\frac{\pi d}{\lambda}\left(\cos (\theta)-\cos \left(\theta_{0}\right)\right)\right)}
\end{aligned}
$$

Normalized to its peak value $\left(A F_{\theta_{0}}\left(\theta_{0}\right)=M\right)$, this implies:

$$
A F_{\theta_{0}}^{n}(\theta)=\frac{\sin \left(\frac{M \pi d}{\lambda}\left(\cos (\theta)-\cos \left(\theta_{0}\right)\right)\right)}{M \sin \left(\frac{\pi d}{\lambda}\left(\cos (\theta)-\cos \left(\theta_{0}\right)\right)\right)}
$$

Comparing (9b) to (12), we can conclude that:

$$
\beta_{k, u}=\left|A F_{\theta_{u}}^{n}\left(\theta_{k}\right)\right|
$$

Based on (13), we can see that: 1) if $\theta_{u} \sim \theta_{k}$, i.e., $\Delta \theta \rightarrow 0$, then $\left|A F_{\theta_{u}}^{n}\left(\theta_{k}\right)\right| \rightarrow 1$. This implies that $\beta_{k, u} \rightarrow 1$, and thus the smaller $\Delta \theta$ is, i.e., $\mathrm{UE}_{k}$ and $\mathrm{UE}_{u}$ are closely at the same direction, the larger $\beta_{k, u}$ is. 2) $A F_{\theta_{u}}^{n}(\theta)$ gives a measure of the 3dB-beamwidth $\Omega_{u}^{3 \mathrm{~dB}}=\left|\theta_{u}-\theta\right|$, which is defined as the angular distance such that $A F_{\theta_{u}}^{n}(\theta)=\sqrt{\frac{1}{2}}$. This implies that $\beta_{k, u}$ implicitly provides information regarding the beamwidth. Therefore, we define the interference threshold $\beta_{0}$, such that $\mathrm{UE}_{k}$ and $\mathrm{UE}_{u}$ with $\beta_{k, u} \geq \beta_{0}$ means that the LOS path of $\mathrm{UE}_{k}$ lies in the $\mathrm{UE}_{u}$ beam. Particularly, the $\beta_{0}$-beamwidth $\Omega_{u}^{\beta_{0}}$ defines the angular distance satisfying $\left|A F_{\theta_{u}}^{n}\left(\theta_{k}\right)\right|=\beta_{0}$. Thus, $\beta$ is an important metric to indicate the level of inter-user interference based on a geometric partial channel knowledge, i.e., $\theta_{k}(\forall k)$.

\section{User Clustering And Power Allocation}

In this section, we propose a low-complex user clustering algorithm based on the proposed geometric interference metric $\beta$. We also present a typical power allocation scheme to investigate the feasibility of NOMA-DBS system. Finally, we derive the NOMA-DBS sum-rate in mono-path channel.

\section{A. User Clustering Method based on $\beta$}

Given the angles of the LOS path of all UEs, we propose our user clustering method. First of all, we need to determine the inter-beam interference threshold $\beta_{0}$ defined in Section III-B. The objective is to regroup UEs two-by-two $\left(\mathrm{UE}_{u}\right.$ and $\mathrm{UE}_{k}$ ) with large inter-beam interference, i.e., high $\beta_{k, u}$ (> $\left.\beta_{0}\right)$, in the same cluster. Then, for the remaining UEs such that $\beta_{k, u}<\beta_{0}$, we assign a unique cluster to each of them with a cluster angle equal to the UE angle, to be served by using only a beam, as indicating in (4).

Since $\beta_{k, u}=\beta_{u, k}$, we create a triangular matrix $\mathbf{D} \in$ $\mathbb{C}^{K-1 \times K}$, indicating the level of inter-beam interference at each $\mathrm{UE}_{k}(\forall k \in\{1 \cdots K\})$ from other UEs $\left(\mathrm{UE}_{u}, \forall u \neq k\right)$, and is expressed as:

$$
\mathbf{D}=\left(\begin{array}{ccccccc}
0 & \beta_{1,2} & \beta_{1,3} & \beta_{1,4} & \cdots & \beta_{1, K-1} & \beta_{1, K} \\
0 & 0 & \beta_{2,3} & \beta_{2,4} & \cdots & \beta_{2, K-1} & \beta_{2, K} \\
0 & 0 & 0 & \beta_{3,4} & \cdots & \beta_{3, K-1} & \beta_{3, K} \\
\vdots & \vdots & \vdots & \vdots & \ddots & \vdots & \vdots \\
0 & 0 & 0 & 0 & \cdots & \beta_{K-2, K-1} & \beta_{K-2, K} \\
0 & 0 & 0 & 0 & \cdots & 0 & \beta_{K-1, K}
\end{array}\right)
$$

We are interested in UEs with $\beta_{k, u} \geq \beta_{0}$. We define an auxiliary matrix $\mathbf{D}_{t}$, where we replace $\beta_{k, u}$ by a negative value e.g., -1 if $\beta_{k, u}<\beta_{0}$. The starting point for the first 2-user cluster is the point $(q, z)$ with the largest value in $\mathbf{D}_{t}$ s.t. $\mathbf{D}_{t}(q, z)>0$, i.e., $\beta_{q, z} \geq \beta_{0}$. Then, we assign one beam $(c=1)$ to $\mathrm{UE}_{q}$ and $\mathrm{UE}_{z}$, with $\theta_{c}=\frac{\theta_{q}+\theta_{z}}{2}$. Subsequently, we remove these two UEs from the set of users to be clustered to prevent their existence in another cluster. After, we compute the same stage until the largest value in $\mathbf{D}_{t}$ is equal to 0 . The proposed user clustering is summarized in Algorithm 1. 


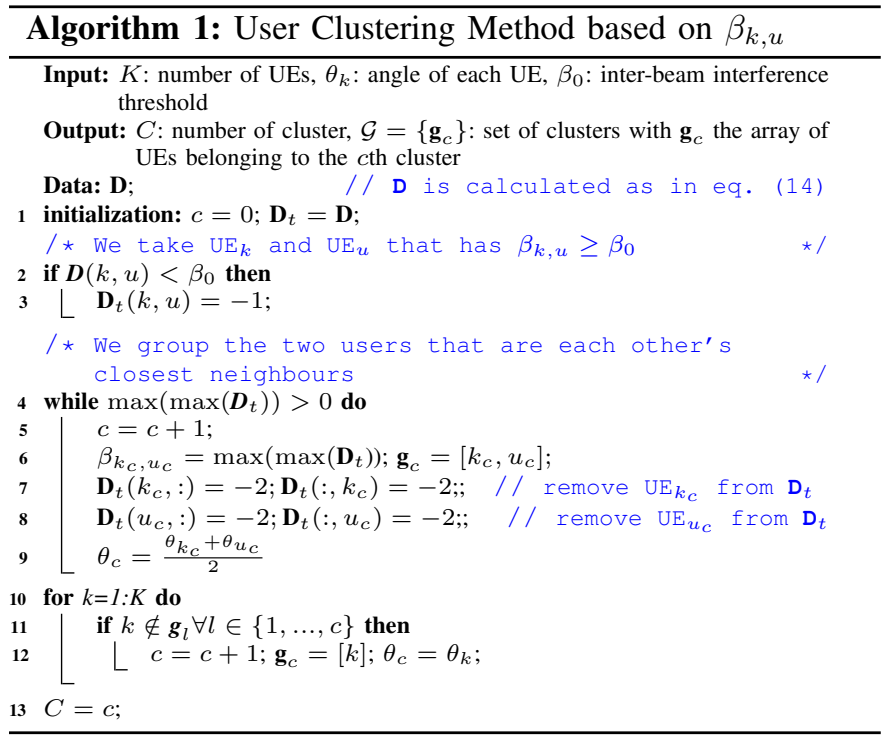

\section{B. Power Allocation Method}

After user clustering, BS applies PD-NOMA for the 2-user cluster, and thus allocates a high power level to the weak UE $\left(\mathrm{UE}_{2, c}\right)$ and a low power level to the strong $\mathrm{UE}\left(\mathrm{UE}_{1, c}\right)$, i.e., $\gamma_{1, c} \leq \gamma_{2, c}$. Many simplified power allocation (PA) methods are used in the literature, typically divided between fixed and dynamic methods. Some of them are based on the UEs' channel gains [16], but all are derived to ensure that the power coefficients of UEs are chosen in inverse order to the order of their channel gains, and that the superimposed signal is transmitted with a unit power in a cluster [16], [17]. Given the cluster set obtained from Section IV-A, we derive a simplified fixed PA scheme to investigate the feasibility of NOMA-DBS system and its performance. This proposed scheme is based on a partial channel knowledge, i.e., the received power of each UE $\left(\left|\mathbf{h}_{l, c} \mathbf{w}_{l, c}\right|^{2}\right)$ and is defined as:

$$
\begin{aligned}
\gamma_{1, c} & =\frac{\left|\mathbf{h}_{2, c} \mathbf{w}_{2, c}\right|^{2}}{\left|\mathbf{h}_{1, c} \mathbf{w}_{1, c}\right|^{2}+\left|\mathbf{h}_{2, c} \mathbf{w}_{2, c}\right|^{2}} \\
\gamma_{2, c} & =\frac{\left|\mathbf{h}_{1, c} \mathbf{w}_{1, c}\right|^{2}}{\left|\mathbf{h}_{1, c} \mathbf{w}_{1, c}\right|^{2}+\left|\mathbf{h}_{2, c} \mathbf{w}_{2, c}\right|^{2}}
\end{aligned}
$$

Based on (15) and (16), the total power constraints for PDNOMA transmission protocol, i.e., $\gamma_{1, c}+\gamma_{2, c}=1$, are performed. Moreover, since $\left|\mathbf{h}_{1, c} \mathbf{w}_{1, c}\right|^{2} \geq\left|\mathbf{h}_{2, c} \mathbf{w}_{2, c}\right|^{2}>0$ and $\gamma_{1, c}+\gamma_{2, c}=1$, then (15) and (16) satisfy the SIC constraint, i.e., $\gamma_{1, c} \leq \gamma_{2, c}$, and also give that $\gamma_{1, c}$ and $\gamma_{2, c}$ are bounded as follows:

$$
\left.\begin{array}{r}
\gamma_{1, c}+\gamma_{2, c}=1 \\
\gamma_{1, c} \leq \gamma_{2, c}
\end{array}\right\} \Longrightarrow\left\{\begin{array}{l}
0<\gamma_{1, c} \leq \frac{1}{2} \\
\frac{1}{2} \leq \gamma_{2, c}<1
\end{array}\right.
$$

\section{Sum-Rate in mono-path channel}

In our work, we adopt the sum-rate $R_{T}$ as the performance metric, which is defined as:

$$
R_{T}=\sum_{k=1}^{K} R_{k}=\sum_{k=1}^{K} \log _{2}\left(1+\operatorname{SINR}_{k}\right) \quad[\mathrm{bps} / \mathrm{Hz}]
$$

where $R_{k}$ and $\mathrm{SINR}_{k}$ are the rate and the signal-tointerference-plus-noise ratio achieved at $\mathrm{UE}_{k}$, respectively.

In mono-path channel, when applying our NOMA-DBS scheme, the SINR of $\mathrm{UE}_{1, c}$ and $\mathrm{UE}_{2, c}$ in a 2-user cluster can be calculated as:

$$
\begin{gathered}
\operatorname{SINR}_{1, c}^{\text {NOMA-DBS }}=\frac{\gamma_{1, c}\left|\mathbf{a}_{1, c}^{H} \mathbf{a}_{c}\right|^{2}}{\sum_{\substack{b=1 \\
b \neq c}}^{C} \sum_{l=1}^{K_{b}} \gamma_{l, b}\left|\mathbf{a}_{1, c}^{H} \mathbf{a}_{b}\right|^{2}+\frac{\sigma_{n}^{2}}{\eta \alpha_{1, c}^{2}}} \\
\operatorname{SINR}_{2, c}^{\text {NOMA-DBS }}=\frac{\gamma_{2, c}\left|\mathbf{a}_{2, c}^{H} \mathbf{a}_{c}\right|^{2}}{\gamma_{1, c}\left|\mathbf{a}_{2, c}^{H} \mathbf{a}_{c}\right|^{2}+\sum_{\substack{b=1 \\
b \neq c}}^{C} \sum_{l=1}^{K_{b}} \gamma_{l, b}\left|\mathbf{a}_{2, c}^{H} \mathbf{a}_{b}\right|^{2}+\frac{\sigma_{n}^{2}}{\eta \alpha_{2, c}^{2}}}
\end{gathered}
$$

For 1-user cluster, the received signal $\mathbf{y}_{1, c}$ and its SINR are also calculated as in (7) and (19), respectively, with $\gamma_{1, c}=1$ and $\mathbf{a}_{c}=\mathbf{a}_{1, c}$.

For the 2-user cluster, the interference factor of UEs belonging to the $c$ th cluster, denoted as $\beta_{c}$, is equal to $\frac{1}{M}\left|\mathbf{a}_{1, c}^{H} \mathbf{a}_{2, c}\right|$, as defined in Section III-B. Since $\mathrm{UE}_{1, c}$ and $\mathrm{UE}_{2, c}$ belong to the same cluster, then $\beta_{c} \geq \beta_{0}$.

For NOMA-DBS, the interference at $\mathrm{UE}_{1, c}$ (strong UE) from $\mathrm{UE}_{2, c}$ (weak UE), denoted as $I_{2 \rightarrow 1}$, is assumed to be totally eliminated by using SIC, i.e., $I_{2 \rightarrow 1}=0$. Moreover, the power received by the cluster's beam with $\theta_{c}=\frac{\theta_{1, c}+\theta_{2, c}}{2}$ at $\mathrm{UE}_{1, c}$ is equal to $\gamma_{1, c}\left|\mathbf{a}_{1, c}^{H} \mathbf{a}_{c}\right|^{2}$, as observed at the numerator in (19). However, $\theta_{1, c} \neq \theta_{c}$, this implies that $\frac{1}{M}<\frac{1}{M}\left|\mathbf{a}_{1, c}^{H} \mathbf{a}_{c}\right|<$ $1\left(=\frac{1}{M}\left|\mathbf{a}_{1, c}^{H} \mathbf{a}_{1, c}\right|\right)$. In addition, from (17), we have $0<\gamma_{1, c} \leq$ $\frac{1}{2}<1$. Accordingly, we obtain $\gamma_{1, c}\left|\mathbf{a}_{1, c}^{H} \mathbf{a}_{c}\right|^{2}<\left|\mathbf{a}_{1, c}^{H} \mathbf{a}_{1, c}\right|^{2}$, where $\left|\mathbf{a}_{1, c}^{H} \mathbf{a}_{1, c}\right|^{2}$ is the received power at $\mathrm{UE}_{1, c}$ in case of DBS only, as shown at the numerator in (3). Therefore, for $\mathrm{UE}_{1, c}$, by passing from DBS to NOMA-DBS, the received power slightly decreases but with no interference from $\mathrm{UE}_{2, c}$. This implies a great performance enhancement for $\mathrm{UE}_{1, c}$.

For $\mathrm{UE}_{2, c}$, as seen in (20), the interference from $\mathrm{UE}_{1, c}$ denoted as $I_{1 \rightarrow 2}$ and equals to $\gamma_{1, c}\left|\mathbf{a}_{2, c}^{H} \mathbf{a}_{c}\right|^{2}$, is not canceled. In addition, the received power using NOMA-DBS is equal to $\gamma_{2, c}\left|\mathbf{a}_{2, c}^{H} \mathbf{a}_{c}\right|^{2}$. If our NOMA-DBS scheme has well grouped the users in order to eliminate the inter-user interference, thus the second term of interference, denoted as $I_{: \rightarrow 2}$ from other clusters is neglected, i.e., $I_{: \rightarrow 2} \rightarrow 0$. Therefore, (20) is simplified to:

$$
\operatorname{SINR}_{2, c}^{\text {NOMA-DBS }} \rightarrow \frac{\gamma_{2, c}\left|\mathbf{a}_{2, c}^{H} \mathbf{a}_{c}\right|^{2}}{\gamma_{1, c}\left|\mathbf{a}_{2, c}^{H} \mathbf{a}_{c}\right|^{2}+\frac{\sigma_{n}^{2}}{\eta \alpha_{2, c}^{2}}}
$$

In the high signal-to-noise ratio regime, (21) can be approximated to $\mathrm{SINR}_{2, c}^{\text {NOMA-DBS }} \rightarrow \frac{\gamma_{2, c}}{\gamma_{1, c}} \geq 1$.

Based on our simplified PA method in Section III-B and as given in (17), we have $0<\gamma_{1, c} \leq \frac{1}{2}, \frac{1}{2} \leq \gamma_{2, c}<1$ and $\gamma_{1, c}+$ $\gamma_{2, c}=1$. If $\mathrm{UE}_{2, c}$ is very weak compared to $\mathrm{UE}_{1, c}$, then $\gamma_{2, c}$ is very large compared to $\gamma_{1, c}$, i.e., $\gamma_{1, c} \rightarrow 0$ and $\gamma_{2, c} \rightarrow 1$. Thus, $\mathrm{SINR}_{2, c}^{\text {NOMA-DBS }} \rightarrow \frac{\gamma_{2, c}}{\gamma_{1, c}}>>1$. In contrast, if their channel gains are comparable, i.e., $\left|\mathbf{h}_{1, c} \mathbf{w}_{1, c}\right|^{2} \rightarrow\left|\mathbf{h}_{2, c} \mathbf{w}_{2, c}\right|^{2}$, then $\gamma_{1, c} \rightarrow \frac{1}{2}$ and $\gamma_{2, c} \rightarrow \frac{1}{2}$. Thus, SINR $_{2, c}^{\text {NOMA-DBS }} \rightarrow \frac{\gamma_{2, c}}{\gamma_{1, c}} \rightarrow 1$ 
and $R_{2, c} \rightarrow 1[\mathrm{bps} / \mathrm{Hz}]$. Therefore, the rate of the weak UE, and consequently the fairness between users strongly depends on the applied PA method. However, for our fixed PA method, the greater the difference between the two users' channel gains, the higher the rate for the weak user, and conversely.

\section{Simulation Results}

The proposed NOMA-DBS scheme, DBS and CB sumrates are compared in mono-path environment, with $K(\in$ $\{2, \cdots, 45\})$ UEs distributed in a cell of radius $100 \mathrm{~m}$. We assume a ULA with $M=64$ at BS. Other system parameters used for performance assessment are listed in Table I. We consider a perfect estimation of angles at the receiver and perfect instantaneous feedback of the received powers $\left|\mathbf{h}_{k} \mathbf{w}_{k}\right|^{2}(\forall k)$ and the estimated angles $\theta_{k}(\forall k)$. The channel features are generated using NYUSIM [14], for instance, the distance and the angle of each user are generated using statistical distributions based on real measurements. We assume that the users belong to the same side of BS, i.e., $0<\theta<180^{\circ}$. The sum-rate is averaged over 4000 channel realizations.

TABLE I: Simulation parameters

\begin{tabular}{||l|l||}
\hline \hline Parameters & Value \\
\hline \hline Carrier frequency & $28[\mathrm{GHz}]$ \\
Channel bandwidth & $20[\mathrm{MHz}]$ \\
Transmission power & $30[\mathrm{dBm}]$ \\
Noise power & $-100.9178[\mathrm{dBm}]$ \\
\hline \hline
\end{tabular}

The system sum-rate of the proposed NOMA-DBS scheme is presented in Fig. 2 as a function of the number of served users, compared with the reference DBS and the CB systems. NOMA-DBS scheme is evaluated with different value of $\beta_{0}$. At TX, DBS needs only the LOS path angle information $\theta_{k}(\forall k)$, while CB needs full channel knowledge, i.e., $\mathbf{H} \in$ $\mathbb{C}^{K \times M}$. This implies that the number of real coefficients needed per channel estimation is $K$ (resp. $2 \times K \times M$ ) for DBS (resp. CB). However, NOMA-DBS requires an additional information relative to DBS, namely the received powers $\left|\mathbf{h}_{k} \mathbf{w}_{k}\right|^{2}(\forall k)$, to apply the classical PA method. Thus, $2 K$ coefficients per channel estimation is needed. In mono-path environment, we have obtained in [4] that DBS outperforms CB. For $\beta_{0} \in\{0.2,0.4,0.6,0.7\}$, we observe that our proposed scheme offers better performance than DBS system, when the number of UEs satisfies $K>6$. As depicted in Fig. 2, when the number of served users increases, the sumrate gain of NOMA-DBS increases with respect to DBS (sumrate gain up to $20 \mathrm{bps} / \mathrm{Hz}$ ). This can be explained by the fact that the interference becomes more severe when the number of UEs increases, affecting the DBS system. In contrast, the NOMA-DBS is applied on more users and the interference effect is reduced. This demonstrates the effectiveness of our proposed method and, in particular, of the user clustering algorithm. More significantly, NOMA-DBS, with a channel overhead $M=64$ times less than that of CB, outperforms CB with a sum-rate gain up to $30 \mathrm{bps} / \mathrm{Hz}$ for 45 users in the cell.

Fig. 3a illustrates the impact of $\beta_{0}$ value on the NOMADBS sum-rate, when $K \in\{20,45\}$ UEs are served si-

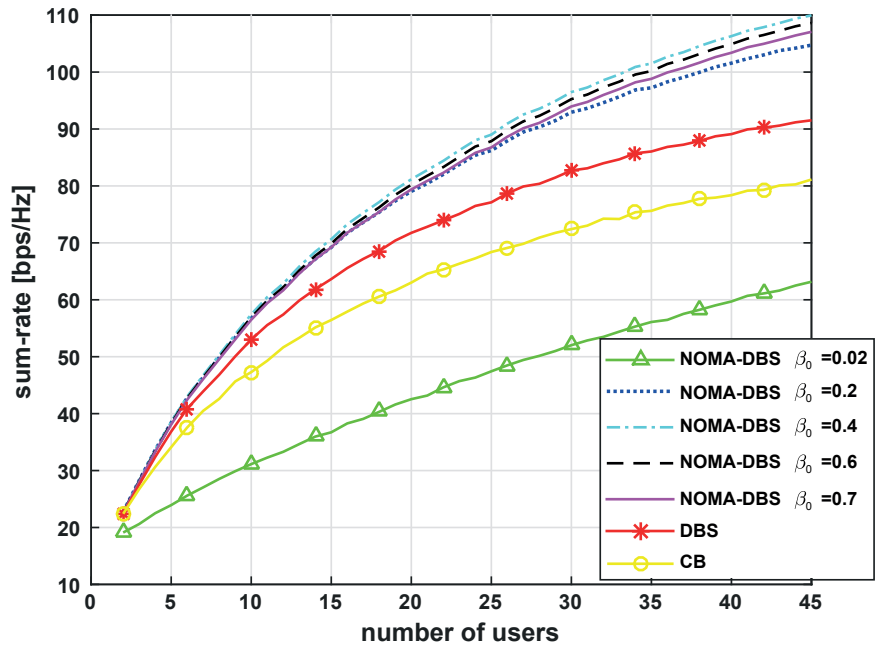

Fig. 2: Sum-rate achieved by the multi-user MIMO system using $\mathrm{CB}$, DBS and NOMA-DBS in a mmWave mono-path environment.

multaneously. Fig. $3 b$ depicts the number of 2-user clusters as a function of $\beta_{0}$, when $K \in\{20,45\}$ UEs are served simultaneously. Firstly, we highlight that NOMA-DBS with $\beta_{0}=1$ is equivalent to DBS. Indeed, Fig. $3 \mathrm{~b}$ shows that the number of 2 -user clusters at $\beta_{0}=1$ is equal to zero. Fig. 3a shows that the sum-rate of NOMA-DBS is greater than that of DBS (or NOMA-DBS with $\beta_{0}=1$ ) for $0.1<\beta_{0}<1$, and have a maximum around $\beta_{0}=0.4$. Moreover, for a wide range of $\beta_{0}$, i.e., $0.2<\beta_{0}<0.7$, NOMA-DBS outperforms DBS and has a comparable sum-rate gain up to $20 \mathrm{bps} / \mathrm{Hz}$ for 45 UEs and $10 \mathrm{bps} / \mathrm{Hz}$ for 20 UEs. This makes the NOMADBS system operational for a large range of $\beta_{0}$ values. It is obvious that the number of 2-user clusters increases when $\beta_{0}$ decreases, as shown in Fig. $3 \mathrm{~b}$.

As shown in Fig. 2, for $\beta_{0} \in\{0.4,0.6,0.7\}$, the smaller $\beta_{0}$ is, the higher the overall sum-rate is achieved using NOMADBS, which is also depicted in Fig. 3a. This can be explained by the fact that when $\beta_{0}$ is small, more UEs are grouped in 2user cluster, as depicted in Fig. 3b, and thus the interference at more UEs is reduced using NOMA-DBS. In contrast, for $\beta_{0}=$ 0.2 , NOMA-DBS achieves better sum-rate compared to DBS but inferior sum-rate with respect to NOMA-DBS with $\beta_{0} \in$ $\{0.4,0.6,0.7\}$. Based on Algorithm 1, the set of the 2-user clusters with $\beta_{0}=0.2$, denoted as $\mathcal{G}$, can be obtained as $\mathcal{G}=$ $\mathcal{G}_{1} \cup \mathcal{G}_{2}$, where $\mathcal{G}_{1}$ is the set of the 2-user clusters with $\beta_{0}=$ 0.4 , and $\mathcal{G}_{2}$ is the set of the 2-user clusters with interference factor $\beta$ s.t. $0.2 \leq \beta<0.4$. The sum-rate degradation of NOMA-DBS with $\beta_{0}=0.2$ with respect to that with $\beta_{0}=0.4$ can be explained by the fact that when UEs belonging to $\mathcal{G}_{2}$ are grouped two-by-two using NOMA-DBS, the power received by the cluster beam at both UEs $\left(\mathrm{UE}_{1, c}, \mathrm{UE}_{2, c}\right)$ is degraded, compared to DBS, due to a large $\Delta \theta=\theta_{l, c}-\theta_{c}(l \in\{1,2\})$.

Besides and as expected, for $\beta_{0}=0.02$, the sum-rate of NOMA-DBS is lower than that of DBS, as illustrated in Fig. 2. Indeed, first, for $\beta_{0}=0.02, \mathrm{UE}_{u}(u \neq k)$ not belonging to the main lobe of $\mathrm{UE}_{k}$ are considered as interferer with $\mathrm{UE}_{k}$. Second, the power received by the cluster beam at both UEs 


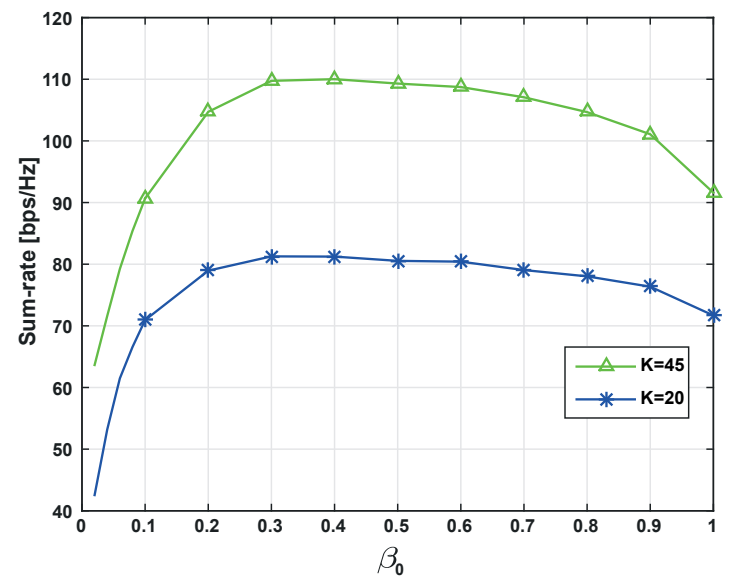

(a) Averaged sum-rate when 20 and 45 users are served simultaneously.

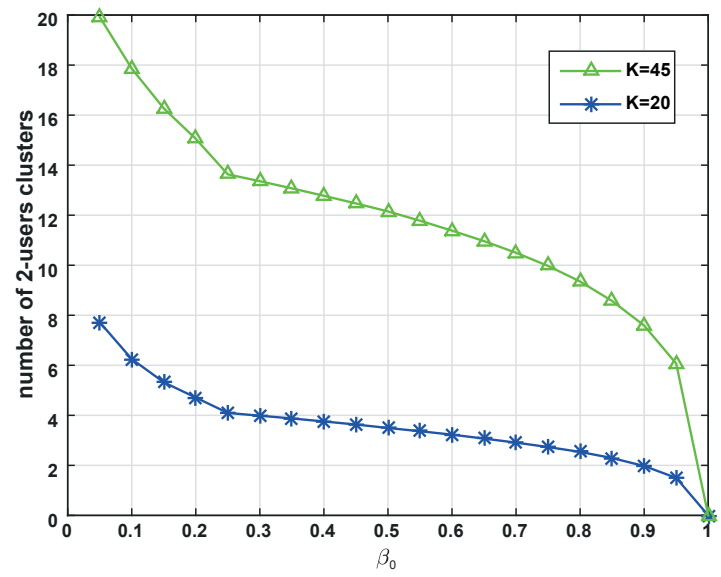

(b) Number of 2-user clusters when 20 and 45 are served simultaneously.

Fig. 3: Impact of the interference threshold $\left(\beta_{0}\right)$ on the performance of the proposed NOMA-DBS scheme.

strongly decreases, since the beam direction is very different than the user direction.

\section{CONCLUSION}

In this paper, we have proposed a joint SDMA and power domain NOMA scheme to resolve the inter-beam interference problem when using multi-user beamsteering, especially in a congested cell. In particular, a two-user clustering algorithm based on a new geometric interference metric is designed, which significantly enhances the system sum-rate, as shown by simulations, e.g., up to $20 \mathrm{bps} / \mathrm{Hz}$ rate gain for 45 users. In our work, the use of a simplified fixed power allocation method enables NOMA-DBS to provide a great overall sumrate compared to DBS, whereas the rate of the weak user strongly depends on the difference between the two users' channel gains.

For future work, a new power allocation scheme based on a partial channel knowledge, that yields to a better fairness between users is needed to be coupled with a generalized multi-user clustering algorithm for NOMA-DBS. To further enhance the NOMA-DBS performance, the value of the interbeam interference threshold $\beta_{0}$ is to be analytically computed.
In this work, we assume that the SIC algorithm completely eliminates the interference $I_{1 \rightarrow 2}=0$. The consideration of small $I_{1 \rightarrow 2} \neq 0$ can be also a perspective of our work.

\section{ACKNOWLEDGMENT}

This work has been funded by IMT Atlantique, Lebanese University research fund program and the AZM association.

\section{REFERENCES}

[1] R. W. Heath, N. Gonzalez-Prelcic, S. Rangan, W. Roh, and A. M. Sayeed, "An overview of signal processing techniques for millimeter wave MIMO systems," IEEE J. Sel. Topics Signal Process., vol. 10, no. 3, pp. 436-453, 2016.

[2] S. Hur, T. Kim, D. J. Love, J. V. Krogmeier, T. A. Thomas, and A. Ghosh, "Millimeter wave beamforming for wireless backhaul and access in small cell networks," IEEE Trans. Commun., vol. 61, no. 10, pp. 4391-4403, 2013.

[3] A. Rozé, M. Hélard, M. Crussière, and C. Langlais, "Millimeter-wave digital beamsteering in highly line-of-sight environments for massive MIMO systems," in Wireless World Research Forum Meeting, vol. 35 , 2015.

[4] I. Khaled, A. El Falou, C. Langlais, B. ElHassan, and M. Jezequel, "Multi-user digital beamforming based on path angle information for mm-wave MIMO systems," in Proc. International ITG Workshop on Smart Antennas (WSA), Feb. 2020.

[5] L. Dai, B. Wang, Y. Yuan, S. Han, I. Chih-Lin, and Z. Wang, "Nonorthogonal multiple access for 5G: solutions, challenges, opportunities, and future research trends," IEEE Commun. Mag., vol. 53, no. 9, pp. $74-81,2015$.

[6] D. Zhang, Z. Zhou, C. Xu, Y. Zhang, J. Rodriguez, and T. Sato, "Capacity analysis of NOMA with mmwave massive MIMO systems," IEEE J. Sel. Areas Commun., vol. 35, no. 7, pp. 1606-1618, 2017.

[7] B. Wang, L. Dai, Z. Wang, N. Ge, and S. Zhou, "Spectrum and energyefficient beamspace MIMO-NOMA for millimeter-wave communications using lens antenna array," IEEE Journal on Selected Areas in Communications, vol. 35, no. 10, pp. 2370-2382, 2017.

[8] S. Liu, C. Zhang, and G. Lyu, "User selection and power schedule for downlink non-orthogonal multiple access (NOMA) system," in Proc. IEEE Int. Conf. Commun. Workshop (ICCW), 2015, pp. 25612565.

[9] M. Zeng, A. Yadav, O. A. Dobre, and H. V. Poor, "Energy-efficient power allocation for MIMO-NOMA with multiple users in a cluster," IEEE Access, vol. 6, pp. 5170-5181, 2018.

[10] X. Zhang, X. Zhu, and H. Zhu, "Joint user clustering and multidimensional resource allocation in downlink MIMO-NOMA networks," IEEE Access, vol. 7, pp. 81 783-81 793, 2019.

[11] X. Chen, F.-K. Gong, G. Li, H. Zhang, and P. Song, "User pairing and pair scheduling in massive MIMO-NOMA systems," IEEE Commun. Lett., vol. 22, no. 4, pp. 788-791, 2017.

[12] Z. Ding and H. V. Poor, "Design of massive-MIMO-NOMA with limited feedback," IEEE Signal Process. Lett., vol. 23, no. 5, pp. 629-633, 2016.

[13] I. Khaled, A. El Falou, C. Langlais, B. ElHassan, and M. Jezequel, "Performance evaluation of linear precoding mmwave multi-user MIMO systems with NYUSIM channel simulator," in 2019 2nd IEEE Middle East and North Africa COMMunications Conference (MENACOMM). IEEE, 2019, pp. 1-6.

[14] M. K. Samimi and T. S. Rappaport, "3-D millimeter-wave statistical channel model for 5G wireless system design," IEEE Trans. Microw. Theory Tech., vol. 64, no. 7, pp. 2207-2225, 2016.

[15] M. El Hassan, A. El Falou, and C. Langlais, "Performance assessment of linear precoding for multi-user massive MIMO systems on a realistic 5G mmWave channel," in 2018 IEEE Middle East and North Africa Communications Conference (MENACOMM). IEEE, 2018, pp. 1-5.

[16] Y. Saito, A. Benjebbour, Y. Kishiyama, and T. Nakamura, "Systemlevel performance evaluation of downlink non-orthogonal multiple access (NOMA)," in Int. Symp. on Personal, Indoor, and Mobile Radio Communications (PIMRC), 2013, pp. 611-615.

[17] T. M. Hoang, X. N. Tran, B. C. Nguyen et al., "Outage analysis of MIMO-NOMA relay system with user clustering and beamforming under imperfect CSI in Nakagami-m fading channels," in Proc. Int. Conf. on Industrial Networks and Intelligent Systems, 2019, pp. 3-17. 